\title{
Estudio morfométrico de las malformaciones craneofaciales experimentales inducidas por ácido retinoico*
}

\author{
T. González ${ }^{1}$, L Lassaletta², L Martínez³, J.A. Tovar, V. Martorell5
}

\begin{abstract}
Resumen: Objetivo: El ácido retinoico es un metabolito activo de la vitamina A que administrado en grandes cantidades tiene efecto teratógeno sobre la embriogénesis de los mamíferos. Hemos investigado los efectos de la exposición temprana de embriones de rata sobre las estructuras craneofaciales.

Diseño: Cuarenta y cinco ratas Sprague-Dawley gestantes fueron tratadas con $125 \mathrm{mg} / \mathrm{kg}$ de ácido all-trans-retinoico el día 10 de gestación. Las 20 ratas controles fueron tratadas con aceite. Los fetos de ambos grupos se extrajeron el día antes de llegar a término y fueron sometidos a un estudio morfológico y otro estudio morfométrico, analizando las malformaciones craneofaciales.

Resultados: Ninguno de los fetos controles presentó malformaciones. El 100\% de los embriones tratados con retinoico presentaron defectos craneofaciales, incluyendo fisuras faciales, exoftalmos, malformaciones e inserción baja de los pabellones auriculares, apéndices faciales y anomalías nasales. El análisis morfométrico reveló un incremento de la distancia entre los poros nasales $(p<0,01)$ y los globos oculares $(p<0,05)$ en los fetos tratados con retinoico. También se encontró una reducción de la distancia lateral del maxilar $(\mathrm{p}<0,01)$ y la mandíbula $(\mathrm{p}<0,01)$.

Conclusiones: Tanto el estudio morfológico como el estudio morfométrico confirman la hipótesis según la cual el ácido retinoico altera el desarrollo craneofacial cuando se administra durante un momento crítico del desarrollo embrionario. Una alteración en la migración de las células de la cresta neural craneal podría ser la razón que explicaría estas anomalías.
\end{abstract}

Palabras clave: Ácido retinoico; Ratas Sprague-Dawley; Embriopatía; Cresta neural; Malformaciones craneofaciales.

Abstract: Objective: Retinoic acid is an active metabolite of Vitamin A that is teratogenic when present in excess during mammalian embriogenesis. We have investigated the effects of early exposure of rat embryos to retinoic acid on craniofacial structures.

Design: Treatment of 45 pregnant Sprague-Dawley rats with $125 \mathrm{mg} . / \mathrm{Kg}$ all-trans-retinoic acid on pregnancy day 10 was performed. Twenty controls were treated only with oil. The fetuses were recovered the day before term, and both morphologic and morphometric analyses of the craniofacial structures were performed.

Results: None of the control fetuses had malformations. Craniofacial defects were observed in $100 \%$ of the retinoic embryos including facial clefts, proptosis, abnormalities and inferior placement of the pinnae, skin tags, and nasal anomalies. Morphometric analyses revealed an increased distance between nasal pores $(p<0,01)$ and between both eyes $(p<0,05)$ in retinoic embryos. A reduced distance of the maxilla $(p<0,01)$ and the mandible $(p<0,01)$ were also noted.

Conclusions: Morphologic and morphometric studies confirm the hypothesis that retinoic acid disturbs normal craniofacial development when administered during a critical period. Hindrance of migration of the cranial neural crest cells may be a main reason to explain these events.

Key words: Retinoic acid; Sprague-Dawley rats; Embriopathy; Neural crest; Craniofacial malformations; Morphometric analysis.

*Este trabajo ha sido financiado en parte con la ayuda del FIS 01/532

1 Médico adjunto. Servicio de Cirugía Maxilofacial

2 Médico adjunto. Servicio de O.R.L

3 Médico adjunto. Departamento de Cirugía Pediátrica

4 Jefe de Departamento. Departamento de Cirugía Pediátrica

5 Jefe de Servicio. Servicio de Cirugía Oral y Maxilofacial

Hospital Universitario La Paz. Madrid, España.

Correspondencia:

T. González Otero

Servicio de Cirugía Maxilofacial

Hospital Universitario La Paz

Paseo de la Castellana 261

28046 Madrid, España.

e-mail: luislassaletta@wanadoo.es

\section{Introducción}

Las malformaciones craneofaciales son las malformaciones más frecuentes en la especie humana. Se han descrito infinidad de síndromes relativos a las estructuras craneofaciales. Entre los más frecuentes en la práctica clínica destacan los síndromes de fisuras orofaciales, como el labio y paladar hendidos, y los síndromes de primer y segundo arcos branquiales, como los síndromes de Franceschetti-Zwahlen-Klein y Treacher-Collins, y la microsomía hemifacial. 
Los retinoides están involucrados en la formación de múltiples órganos durante la embriogénesis. Sin embargo, la exposición del embrión a dosis elevadas de retinoides durante las primeras semanas del desarrollo embrionario puede ocasionar un aborto espontáneo o graves defectos congénitos., ${ }^{1,2}$ Las malformaciones que se producen en la embriopatía por vitamina A y sus derivados (ácido retinoico) son bien conocidas en humanos. Estas malformaciones se producen en estructuras derivadas de la cresta neural como el esqueleto craneofacial, el timo y el corazón. A raíz de estos hallazgos, se empezaron a usar los retinoides en modelos de experimentación animal, siendo actualmente los agentes teratógenos más ampliamente estudiados como inductores de malformaciones craneofaciales en animales de laboratorio. Se ha propuesto que las malformaciones craneofaciales se producen por una alteración en la migración y distribución de las células de la cresta neural craneal en los primordios craneofaciales, causada por el ácido retinoico. ${ }^{3}$ Sin embargo, aún no existen estudios morfométricos que analicen con detalle las alteraciones craneofaciales de los embriones expuestos a ácido retinoico.

El objetivo de este trabajo es demostrar que la exposición de ratas gestantes al ácido retinoico induce malformaciones craneofaciales a sus embriones, y realizar un estudio morfométrico, con el objeto de demostrar que las medidas craneofaciales de los embriones expuestos a ácido retinoico se encuentran alteradas con relación a las medidas craneofaciales de embriones de rata controles.

\section{Material y método}

Se emplearon ratas Sprague-Dawley hembras vírgenes de 220-250 gramos de peso y machos de fertilidad probada. Los animales fueron alojados en las instalaciones del laboratorio experimental del Hospital La Paz de Madrid, España y fueron alimentados con el granulado especial para rata, y agua de bebida sad libitum». Su manejo se ajustó a las exigencias de la Unión Europea vigentes (E.C. 86/L609) y a las recogidas en el R.D. 223/1988. En el grupo del ácido retinoico las hembras recibieron una sola dosis de $125 \mathrm{mg} / \mathrm{Kg}$ de ácido all-trans-retinoico diluido en aceite de oliva a una dilución de $40 \mathrm{mg} / \mathrm{ml}$, administrado mediante una sonda nasogástrica el día 10 de gestación. Los animales del grupo control recibieron una dosis de $125 \mathrm{mg} / \mathrm{Kg}$ de aceite de oliva por sonda nasogástrica en el día 9,5 de gestación. El día cero de la gestación fue contado a partir del momento en que se demostró la presencia de espermatozoides en el frotis vaginal. La obtención de los embriones se realizó mediante cesárea el día 21 bajo anestesia general (el embrión es a término con 22 días). Para la selección de los animales del grupo control se eligieron aleatoriamente 4 animales por camada, mientras que para la selección de los embriones del grupo retinoico se eligieron aleatoriamente 5 animales por camada. Los animales seleccionados de los dos grupos fueron fijados en formol al 10\% durante 48-72 horas. A continuación se realizó el estudio macroscópico de las estructuras craneofaciales.

Las variables analizadas fueron las siguientes: existencia y tipo de fisuras faciales (maxilares, mandibulares o ambas), presencia de exoftalmos en cada lado, ausencia de bigotes en cada lado, implantación normal o baja de ambos pabellones auriculares, malformaciones de pabellón auricular (anotia, microtia o dismorfia), presencia y posición de apéndices faciales y deformidades nasales (puente nasal deprimido, giba aumentada o narinas antevertidas).

El estudio morfométrico de las cabezas de los fetos se realizó con el programa Adobe Photoshop 6.0 para Windows, empleando la herramienta «medición»que permite calcular en píxeles la distancia entre 2 puntos cualesquiera de una imagen. Las figuras 1 y 2 muestran las variables cuantitativas consideradas en las visiones frontal y lateral, que fueron: la longitud del proceso maxilar, la distancia entre los poros nasales, la distancia entre los globos oculares, la longitud antero-posterior del proceso maxilar, la longitud anteroposterior de la mandíbula, la distancia entre el poro nasal y el pabellón y la distancia entre el punto cefálico más alto y el pabellón.

Para evitar el posible sesgo que pudieran ocasionar las diferencias entre las medidas faciales de los 2 grupos sobre cada medida en particular, la longitud de cada variable cuantitativa se dividió entre la longitud media paralela de su grupo correspondiente. Por ejemplo, la medida de los maxilares en la visión frontal podría estar sesgada por la medida máxima derecha -izquierda o medida transversal de la cara. Dicho de otro modo, es posible que el maxilar del feto malformado fuera más corto que el del feto del grupo control porque la longitud transversal de la cara del feto malformado fuera más estrecha que la del feto control y no porque realmente el maxilar fuera más corto. Para evitar este posible sesgo, dividimos la distancia del maxilar entre la distancia transversal de la cara tanto en el feto malformado como en el feto control.

De este modo, para el posterior análisis estadístico, en lugar de comparar las longitudes crudas de cada variable cuantitativa hemos comparado la longitud corregida, calculada dividiendo la longitud cruda entre el diámetro medio correspondiente en función de la medida (longitud transversal, longitud antero-posterior o longitud cráneo-caudal), según cada caso.

Así, la longitud de los procesos maxilares derecho e izquierdo, la distancia entre los poros nasales y la distancia entre los globos oculares se expresó en \% con relación a la longitud transversal de la cara (Fig. 


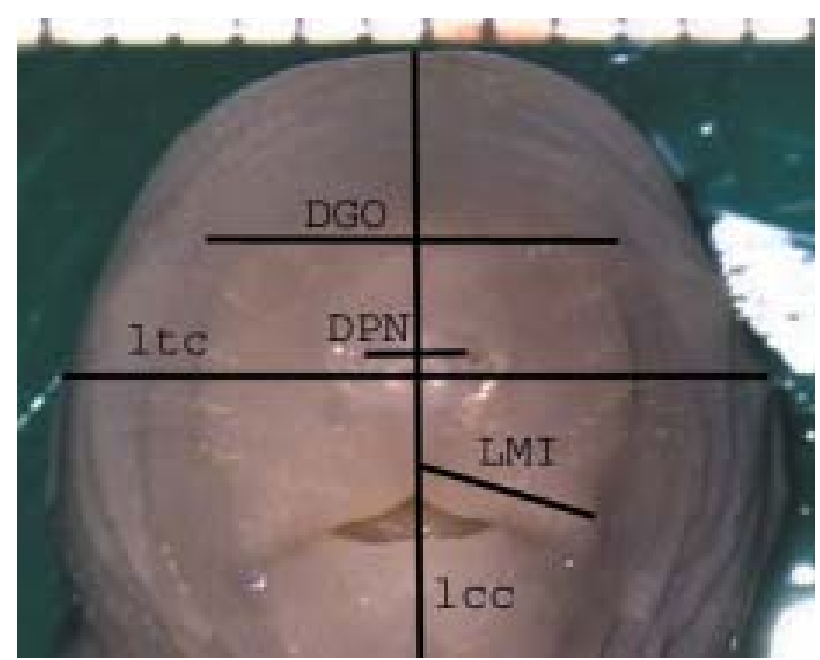

Figura 1. Visión frontal de un feto control. Las variables cuantitativas representadas son LMI: Longitud del maxilar izquierdo, DPN: Distancia entre los poros nasales y DGO: Distancia entre los globos oculares. Itc representa la longitud transversal de la cara, mientras que lcc es la longitud cráneo-caudal.

1); la distancia antero-posterior del maxilar, la distancia antero-posterior de la mandíbula y la distancia poro nasal-pabellón auricular se expresaron en \% con relación a la longitud antero-posterior de la cara; y la distancia punto cefálico superior-pabellón auricular se expresó en \% con relación a la longitud cráneo-caudal de la cara (Fig. 2). La comparación de las medias corregidas entre los grupos se realizó empleando el test no paramétrico de U Mann-Whitney. Se consideró un nivel de significación $\mathrm{p}<0,05$.

\section{Resultados}

Ninguno de los fetos controles presentó alteraciones de las variables estudiadas. Los fetos tratados con retinoico presentaron numerosas malformaciones craneofaciales. El 57,8\% de los fetos tratados con retinoico presentaron fisuras faciales y el $78 \%$ presentaron exoftalmos. Al 20\% de los fetos tratados con retinoico les faltaban los bigotes en el lado derecho de la cara y al 13\% en el lado izquierdo. El 90\% de los fetos tratados con retinoico tuvieron pabellones auriculares de implantación baja en el lado derecho y el $86 \%$ en el izquierdo. De los fetos expuestos al retinoico, el $64,4 \%$ presentaban malformaciones del pabellón auricular derecho y el $64 \%$ del pabellón izquierdo. El 60\% de los fetos tratados con retinoico tuvieron apéndices faciales en el lado derecho y el $64,4 \%$ en el lado izquierdo. El 55\% de los fetos tratados con retinoico presentaron algún tipo de deformidad nasal.

La tabla 1 muestra un resumen del análisis morfométrico de las variables cuantitativas. Al realizar el estudio morfométrico, observamos que la longitud del proceso maxilar derecho de los fetos tratados con reti-

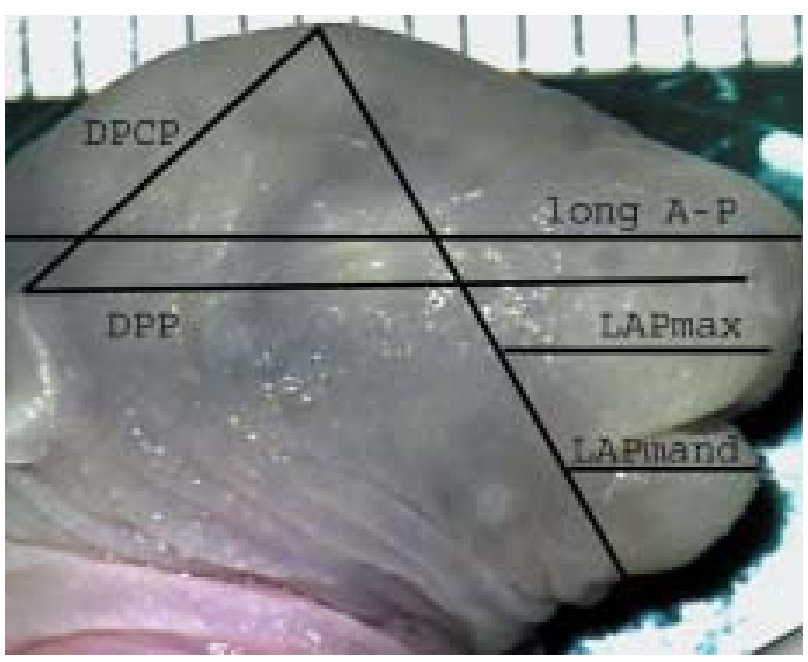

Figura 2. Visión lateral derecha de un feto control. Las variables cuantitativas representadas son DPCP: distancia entre el punto cefálico superior y el pabellón auricular, DPP: distancia poro nasal pabellón auricular, LAP max: longitud antero-posterior del maxilar, LAP man: longitud antero-posterior de la mandíbula. long A-P corresponde a la longitud antero-posterior de la cara.

noico fue menor que la de los fetos controles, si bien la diferencia no fue estadísticamente significativa, y lo mismo ocurrió con la longitud del proceso maxilar izquierdo. Por otra parte, en el plano frontal, tanto la distancia entre los poros nasales como la distancia entre los globos oculares de los fetos tratados con retinoico fue mayor que la de los fetos controles, siendo la diferencia estadísticamente significativa (Fig. 3).

En el plano lateral, la distancia anteroposterior del maxilar y la distancia anteroposterior de la mandíbula de los fetos tratados con retinoico fue menor que la de los fetos controles, siendo la diferencia estadísticamente significativa. Al evaluar las distancias que valoran la posición del pabellón auricular observamos que la distancia entre el poro nasal derecho y el pabellón auricular derecho de los fetos tratados con retinoico fue menor que la de los fetos controles, siendo la diferencia estadísticamente significativa, y lo mismo ocurrió al evaluar el lado izquierdo. Igualmente, la distancia entre el punto cefálico superior y los pabellones auriculares derecho e izquierdo de los fetos tratados con retinoico fue mayor que la de los fetos controles, siendo la diferencia estadísticamente significativa.

\section{Discusión}

A partir del descubrimiento de la asociación entre el uso de los retinoides y las malformaciones en los embriones humanos comenzó a usarse el ácido retinoico en modelos de experimentación animal. Webster y cols, ${ }^{1}$ realizaron una serie de estudios en animales de laboratorio administrando Accutane ${ }^{\circledR}$ a rato- 
Tabla 1. Análisis morfométrico. Variables cuantitativas control vs. ácido retinoico

\begin{tabular}{|c|c|c|c|c|c|c|}
\hline Variable & $\mathbf{N}$ & $\begin{array}{l}\text { Media cruda } \\
(\mathbf{m m})\end{array}$ & $\begin{array}{c}\text { Media } \\
\text { correctora }(\mathrm{mm})\end{array}$ & $\begin{array}{c}\text { Media } \\
\text { corregida (\%) }\end{array}$ & $\begin{array}{l}\text { Desviación } \\
\text { estandar }\end{array}$ & $\mathbf{P}$ \\
\hline \multicolumn{7}{|c|}{ Longitud del maxilar } \\
\hline Control & 20 & 3,01 & 10,96 & 27,5 & 1,1 & 0,451 \\
\hline Retinoico & 45 & 2,17 & 8,34 & 26 & 6,9 & \\
\hline \multicolumn{7}{|c|}{ Longitud del maxilar izdo. } \\
\hline Control & 20 & 2,97 & 10,96 & 27,1 & 1,6 & 0,765 \\
\hline Retinoico & 45 & 2,22 & 8,34 & 26,6 & 6,2 & \\
\hline \multicolumn{7}{|c|}{ Distancia entre los poros nasales } \\
\hline Control & 20 & 1,64 & 10,96 & 14,9 & 1,3 & $<0,0001$ \\
\hline Retinoico & 45 & 1,55 & 8,34 & 18,6 & 2,7 & \\
\hline \multicolumn{7}{|c|}{ Distancia entre los globos oculares } \\
\hline Control & 20 & 7,55 & 10,96 & 68,9 & 3,9 & 0,041 \\
\hline Retinoico & 45 & 6,13 & 8,34 & 73,6 & 9,8 & \\
\hline \multicolumn{7}{|c|}{ Longitud anteroposterior del maxilar } \\
\hline Control & 20 & 6,34 & 16,61 & 38,2 & 2 & $<0,0001$ \\
\hline Retinoico & 45 & 4,23 & 13,52 & 31,3 & 5,1 & \\
\hline \multicolumn{7}{|c|}{ Longitud anteroposterior de la mandibula } \\
\hline Control & 20 & 4,83 & 16,61 & 29,1 & 2,4 & 0,001 \\
\hline Retinoico & 45 & 3,33 & 13,52 & 24,6 & 4,7 & \\
\hline \multicolumn{7}{|c|}{ Distancia poro nasal-Pabellón derecho } \\
\hline Control & 20 & 13,66 & 16,61 & 82,2 & 2,6 & $<0,0001$ \\
\hline Retinoico & 45 & 8,6 & 13,52 & 63,5 & 23,3 & \\
\hline \multicolumn{7}{|c|}{ Distancia poro nasal-Pabellón Izquierdo } \\
\hline Control & 20 & 13,41 & 16,61 & 80,7 & 3,4 & $<0,0001$ \\
\hline Retinoico & 45 & 7,73 & 13,52 & 57,1 & 27,8 & \\
\hline \multicolumn{7}{|c|}{ Distancia punto cefálico- Pabellón derecho } \\
\hline Control & 20 & 6,11 & 11,09 & 55,1 & 4,9 & 0,003 \\
\hline Retinoico & 45 & 5,23 & 9,22 & 56,8 & 20,6 & \\
\hline \multicolumn{7}{|c|}{ Distancia punto cefálico- Pabellón izquierdo } \\
\hline Control & 20 & 5,62 & 11,09 & 50,7 & 5,2 & 0,002 \\
\hline Retinoico & 45 & 4,84 & 9,22 & 52,5 & 25,1 & \\
\hline
\end{tabular}

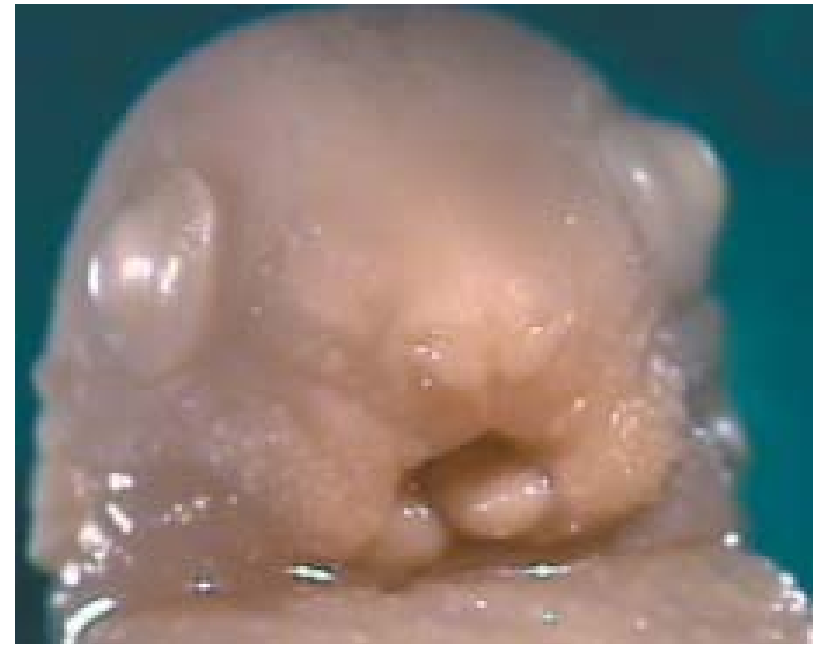

Figura 3. Feto tratado con retinoico que presenta una fisura mandibular, exoftalmos bilateral y un aumento de la distancia entre los poros nasales.

nes durante la embriogénesis precoz. Indujeron malformaciones en el oído externo, hipoplasia maxilar y mandibular, fisura palatina y defectos cardíacos y del timo, es decir, malformaciones de estructuras derivadas de la cresta neural. Granström y cols. en 1990,4,5 vieron como la administración de $15 \mathrm{mg} / \mathrm{Kg}$ de etretinato, o bien de $60 \mathrm{mg} / \mathrm{Kg}$ de ácido retinoico a ratas Sprague Dawley producía muerte fetal. La administración de $10 \mathrm{mg} / \mathrm{Kg}$ de etretinato o bien $40 \mathrm{mg} / \mathrm{Kg}$ de ácido retinoico producía malformaciones menos severas, pudiendo llegar los fetos a término. Cuando los retinoides eran administrados precozmente en la gestación, días 7-8, se producían exencefalia y defectos del cierre del tubo neural. Si los retinoides se administraban más tarde, días 10-11, se producían anomalías digitales y en la cola. Cuando los retinoides se administraban entre los días 8,5 y 9, se inducía un síndrome muy similar al de los arcos branquiales humanos. La estructura aislada que más claramente estaba afectada por el retinoico era la cresta neural craneal. Como puede observarse, el día del desarrollo embriológico en el que se administra el teratógeno es fundamental, puesto que el desarrollo embriológico es un proceso dinámico. ${ }^{3}$ Nosotros administramos el teratógeno las primeras horas del día 10 de gestación y las malformaciones aparecen 
en tejidos derivados del proceso frontonasal de los arcos branquiales y por tanto tejidos en cuyo desarrollo juegan un papel crucial las células de la cresta neural craneal.

Tras la administración del teratógeno se produce un retraso considerable en la morfogénesis facial que se manifiesta en la disminución de la longitud de varias distancias faciales y que medimos en el estudio morfométrico. La longitud de los procesos maxilares, tanto derecho como izquierdo fueron menores en los fetos tratados con retinoico que en los fetos controles, si bien este dato no fue estadísticamente significativo. Aunque no hemos encontrado referencias a estas medidas en concreto en la literatura, la disminución del tamaño podría explicarse por el desarrollo anormal del maxilar. La distancia entre los poros nasales de los fetos tratados con retinoico fue significativamente mayor que la de los fetos controles. Curiosamente, en el estudio de Granström y Kullaa-Mikkonen, ${ }^{5}$ esta distancia estaba disminuida en lugar de aumentada, y los autores lo atribuyen a la disminución de la anchura nasal. En nuestro estudio, cuando consideramos la distancia cruda (sin factor de corrección) entre los poros nasales, ésta era menor en los fetos con retinoico. Sin embargo, al aplicar el factor de corrección, en este caso la longitud transversal de la cara, la distancia entre los poros nasales fue mayor en los fetos con retinoico, siendo además estadísticamente significativa. En el citado estudio, ${ }^{5}$ no se especifica si se ha aplicado o no una corrección en relación con la disminución global del tamaño de la cara. Nosotros explicamos el aumento de la distancia entre los poros por la tendencia que existe en los fetos tratados con el retinoico al retraso en la fusión de los diferentes procesos faciales durante le embriogénesis y que se manifiesta claramente en la formación de las fisuras faciales, de tal forma que un retraso en la fusión de los procesos nasales se manifestaría tanto en un aumento de la distancia entre los poros nasales como en la aparición de fisuras o fístulas.

La distancia entre los globos oculares de los fetos tratados con retinoico fue mayor que la de los fetos controles, siendo la diferencia estadísticamente significativa. Este dato se puede relacionar con la tendencia al hipertelorismo que existe en los síndromes humanos derivados de alteraciones de las células de la cresta neural. Sin embargo, debe tenerse en cuenta que la mayor parte de los fetos expuestos a retinoico presentaban exoftalmos, hallazgo que también expresa la alteración en la morfogénesis. Por ello, en los fetos con exoftalmos se midió la distancia entre los globos oculares exoftálmicos, mientras que en los fetos sin exoftalmos, bien tratados con retinoico o bien controles, al estar fusionados los párpados, medimos las distancias entre los cantos intemos de las hendiduras palpebrales. La longitud ántero-posterior del maxilar y la lon- gitud ántero-posterior de la mandíbula fueron menores en los fetos tratados con retinoico comparadas con las mismas medidas en los fetos controles, siendo la diferencia estadísticamente significativa. Con estas dos medidas hemos corroborado los hallazgos de Granström y Kullaa-Mikkonen ${ }^{6}$ que simplemente describen como sus fetos tratados con etretinato pueden tener la cara aplanada, sin aportar ningún tipo de medida. Del mismo modo Webster y cols, ${ }^{1}$ describieron hipoplasia del maxilar y la mandíbula en ratones tratados con Accutane $^{\circledR}$. La explicación de estos hallazgos sería también el retraso de morfogénesis facial.

En nuestro estudio la posición de los pabellones auriculares se valoró midiendo la distancia desde el poro nasal hasta el pabellón auricular, y la distancia entre el punto cefálico superior y el pabellón auricular, considerando ambos pabellones por separado. Los pabellones de los fetos tratados con retinoico se localizaron más anterior e inferiormente, comparados con los pabellones de los fetos controles, siendo ambos hallazgos estadísticamente significativos para ambos lados. Del mismo modo, en el estudio descriptivo se valoró la implantación normal o baja de cada pabellón auricular, siendo baja en el 86\% y el 90\% de los fetos tratados con retinoico, en el lado derecho e izquierdo respectivamente. En el estudio de Granström y cols, ${ }^{4}$ en los fetos tratados con retinoico los pabellones eran más inferiores pero más posteriores. Estos autores describen la posición de los pabellones sin emplear ningún tipo de medición, por lo que el resultado no es comparable al nuestro.

En función de los resultados obtenidos en nuestro estudio, compartimos la idea de que el origen de las malformaciones craneofaciales está en la alteración de las células de la cresta neural craneal. Esta teoría fue inicialmente propuesta por Mc Credie, 7,8 en la embriopatía por talidomida y más tarde ampliamente defendida por Jhonston, 9,10 en la teratogénesis de la hipervitaminosis A. Se considera que existe un déficit en el mesénquima del proceso frontonasal y de los arcos branquiales como resultado de un fallo en el proceso de migración de las células de la cresta neural craneal y de su contribución a los primordios faciales. Esta teoría es la más aceptada en el momento actual y existen múltiples estudios que la apoyan. ${ }^{4,11-13}$

\section{Conclusiones}

En conclusión, la exposición de ratas Sprague-Dawley al ácido retinoico en el día diez de gestación induce importantes malformaciones craneofaciales en sus embriones, probablemente ocasionadas por una alteración en la migración de las células de la cresta neural craneal a los primordios faciales en desarrollo, es decir proceso frontonasal y arcos branquiales. Estas 
malformaciones no sólo se observan en el estudio descriptivo de los embriones, sino que alteran sus medidas craneofaciales, como se pone de manifiesto en el estudio morfométrico.

\section{Bibliografía}

1. Webster WS, Johnston MC, Lammer EJ, Sulik KK. Isotretinoin embryopathy and the cranial neural crest: an in vivo and in vitro study. J Craniofac Genet Dev Biol 1986;6:211-22.

2. Geiger JM, Baudin M, Saurat JH. Teratogenic risk with etretinate and acitretin treatment. Dermatology 1994; 189:109-16.

3. Johnston MC. The neural crest in abnormalities of the face and brain. Birth Defects 1975;11:1.

4. Granström G. Retinoid-induced ear malformations. Otolaryngol Head Neck Surg 1990;103:702-9.

5. Granström G, Kullaa-Mikkonen A. Experimental craniofacial malformations induced by retinoids and resembling branchial arch syndromes. Scand J Plast Reconstr Surg Hand Surg 1990;24:3-12.
6. Granström G, Kullaa-Mikkonen A. Retinoid-induced nasal malformations. ORLJ Otorhinolaryngol Relat Spec 1990; 52:239-48.

7. McCredie J. Thalidomide and congenital Charcot's joints. Lancet 1973;2:1058-61.

8. McCredie J. Embryonic neuropathy. A hypothesis of neural chest injury as the pathogenesis of congenital malformations. Med J Aust 1974;1:159-63.

9. Johnston MC. The neural crest in abnormalities of the face and brain. Birth Defects 1975;11:1.

10. Johnston MC, Morris GM, Kushner DC. Abnormal organogenesis of facial structures. In: Wilson $\mathrm{M}$ and Fraser G, eds. Handbook of Teratology, Mechanisms and pathogenesis. New York: Plenum Press, 1977;421.

11. Poswillo D. The pathogenesis of the first and second branchial arch syndrome. Oral Surg Oral Med Oral Pathol 1973;35:302-28.

12. Poswillo D. The pathogenesis of the Treacher Collins syndrome (mandibulofacial dysostosis). Br J Oral Surg 1975; 13:1-26.

13. Hassel JR, Greenberg JH, Johnston MC. Inhibition of cranial neural crest cell development by vitamin A in cultured chick embryo. J Embryol Exp Morphol 1977;39:267-71. 